\title{
Lições Aprendidas de Uso de Baixa Tecnologia em uma Disciplina Engenharia de Software Aplicando Diversas Metodologias Ativas: Um Relato de Experiência
}

\author{
Yandson Costa ${ }^{1}$, Sebastião Santos ${ }^{1}$, Nathasha Pinto ${ }^{1}$, Davi Viana ${ }^{2}$, Luis Rivero ${ }^{2}$ \\ ${ }^{1}$ Departamento de Informática - DEINF \\ Universidade Federal do Maranhão - UFMA \\ ${ }^{2}$ Programa de Pós-Graduação em Ciência da Computação - PPGCC \\ Universidade Federal do Maranhão - UFMA \\ \{yandson.jesus, sebastiaohns, nathashaara\}lgmail.com, \\ davi.viana@lsdi.ufma.br, luisrivero@nca.ufma.br
}

\begin{abstract}
Software Engineering (SE) is an area of computing, whose objective is to support IT professionals in the development of quality software in an economical way. Applying different teaching methodologies is essential to meet the expectations of different audiences during the process of learning $S E$ content. This paper presents an experience report of the application of different low-tech learning objects and active methodologies during an SE teaching discipline in order to include practical activities in the teaching process. At the end of the course, students evaluated the methodologies and artifacts used, indicating positive and negative aspects of each one. The lessons learned and the attributes that impact the acceptance of the adopted teaching methodologies were identified, such as: interaction between students, engagement and relationship of the topics taught with the industry.
\end{abstract}

Resumo. A Engenharia de Software (ES) é uma área da computação, cujo objetivo é apoiar profissionais de TI no desenvolvimento de software de qualidade de forma econômica. Aplicar distintas metodologias de ensino é fundamental para atender as expectativas de diversos públicos durante o processo de aprendizagem dos conteúdos da ES. Este trabalho apresenta um relato de experiência da aplicação de distintos objetos de aprendizagem de baixa tecnologia e metodologias ativas durante uma disciplina de ensino de ES com o objetivo de incluir atividades práticas no processo de ensino. Ao final da disciplina, os alunos avaliaram as metodologias e os artefatos utilizados, indicando aspectos positivos e negativos de cada um. Foram identificadas as lições aprendidas e os atributos que impactam na aceitação das metodologias de ensino adotadas, como: interação entre os discentes, engajamento e relação dos tópicos ensinados com a indústria.

\section{Introdução}

A Engenharia de Software (ES) descreve todos os conhecimentos da produção de software [Humphrey, 1998], abrangendo processos, métodos de gerenciamento e produção de software, bem como variadas opções de técnicas e ferramentas. Esses conhecimentos possibilitam que os profissionais desenvolvam seus produtos de 
software de maneira adequada [Santos et al., 2012]. Por envolver diversos conhecimentos, é necessário um esforço adicional para compartilhá-los com os discentes em cursos de graduação ou pós-graduação, tornando-se uma área de estudo com disciplinas desafiadoras [Andrade et al., 2017].

O ensino da ES está presente em grande parte dos cursos de computação no Brasil e no mundo [Zorzo et al., 2017]. Currículos de referência sugerem a inclusão da ES devido à sua importância para a formação de profissionais com conhecimentos tanto acadêmicos quanto alinhados às demandas do mercado [Wangenheim e Silva, 2009]. Assim, nas disciplinas de ES, o professor apresenta conceitos teóricos através de aulas expositivas e aulas de laboratório, além de realizar discussões de casos práticos da área [Lemos et al., 2019].

Devido à quantidade de conhecimentos presentes na ES, muitos discentes de cursos de computação ainda encontram dificuldades no aprendizado de ES [Cunha et al., 2018]. Existem relatos de insatisfação por parte dos discentes com relação ao uso de metodologias de ensino predominantemente teóricas e com poucas atividades práticas [Mendes et al., 2019]. Recentemente, houve um crescimento na utilização de diferentes metodologias no ensino da ES, como pode ser observado nos resultados do trabalho de Cunha et al. (2018), que realizou uma revisão sistemática reunindo 113 trabalhos que relatam a criação e/ou uso de diversos objetos de aprendizagem e metodologias para apoiar o ensino de ES. A aplicação prática dos conceitos com diversas metodologias e uso de baixa tecnologia (sem o uso de computador) pode ser útil no processo de ensino de ES, trazendo vários benefícios, como [Lima et al., 2019]: elevação do nível de aprendizado; elevação do nível de motivação; elevação do desenvolvimento profissional e tomada de decisões; elevação do índice de notas e prioridade da turma; valorização das habilidades interpessoais e percepção; promoção de atividades que estimulam o pensamento crítico e sistemático; adoção de habilidades de experiências relacionadas à prática; modelo de ensino e aprendizagem a ser seguido; adoção de inovações relacionadas a soluções técnicas; adoção do desenvolvimento de análises, síntese e conceitualizarão; diminuição do índice de desistência e de faltas; aprimoramento da relação entre o aluno com o professor; e auxílio na complexidade dos conteúdos abordados.

Motivados pela expectativa dos alunos e da criação e disponibilização de novos objetos e ferramentas de apoio ao ensino, este trabalho apresenta um relato de experiência da aplicação de distintas metodologias em sala de aula para o ensino de ES e os objetos de aprendizagem de baixa tecnologia associados às metodologias. Foi avaliada a percepção dos discentes sobre as metodologias e objetos de aprendizagem aplicados através do uso de um questionário adaptado e a aplicação de uma dinâmica de Focus Group - Lovers VS Haters. Os resultados podem servir como base para que futuras disciplinas de ES adotem metodologias de ensino diversificadas e que possam ser analisados os pontos positivos e negativos decorrentes da sua aplicação.

\section{Trabalhos Relacionados}

O ensino da ES está presente em grande parte dos cursos de computação no Brasil e no mundo [Zorzo et al., 2017] [ACM/IEEE, 2013], tendo fundamental importância na formação de profissionais com conhecimentos tanto acadêmicos quanto alinhados às demandas do mercado [Wangenheim e Silva, 2009]. Assim, nas disciplinas de ES, o 
professor pode apresentar conceitos teóricos, além de executar práticas e realizar discussões de estudo de caso da área [Lemos et al., 2019]. Nesse contexto, diversas iniciativas visam melhorar a educação em ES. Essas iniciativas utilizam diferentes metodologias de ensino que aproximam ou simulam as atividades da indústria. $\mathrm{Na}$ aplicação de metodologias ativas para o ensino de ES observa-se que a maioria dos trabalhos relatam a utilização de jogos como objetos de aprendizagem [Cunha et al., 2018]. Por exemplo, Araujo et al. (2017) aplicaram o jogo BlackBox como meio de reforçar os conteúdos ministrados em relação ao teste funcional de software. Além disso, o jogo foi avaliado quanto aos seus aspectos educacionais, experiência do usuário e aprendizado sob a perspectiva dos alunos.

Outros trabalhos visam identificar o grau de satisfação dos discentes com as metodologias de ensino adotadas. Por exemplo, Mendes et al. (2019) identificaram que os alunos estão insatisfeitos com metodologias de ensino predominantemente teóricas e com poucas atividades práticas. Este trabalho foi realizado no contexto do curso de Ciência da Computação do Estado do Maranhão com discentes que tinham cursado a disciplina de ES e discentes que ainda não tinham cursado a disciplina, visando obter informações sobre as expectativas e opiniões dos discentes, antes e depois da sua participação no processo de ensino. Nesse trabalho, os alunos relataram que gostariam que a disciplina fosse voltada para a aplicação prática dos conceitos e tivesse uma abordagem utilizando diferentes materiais de apoio.

Apesar do enfoque em jogos educacionais e das expectativas dos discentes, também há a necessidade de estudos que foquem na aplicação de metodologias de ensino diferentes das tradicionais [Santos et al., 2014]. Por exemplo, no trabalho de Andrade et al. (2015) é apresentada a evolução de uma metodologia de ensino, que foca em permitir uma maior interação dos alunos com atividades reais encontradas no mercado, através da realização de atividades práticas alinhadas às aulas teóricas e uma interação com profissionais atuantes no mercado de trabalho e pesquisadores da área. Os autores desse trabalho avaliaram a metodologia como um todo e cada objeto de aprendizagem utilizado, identificando a satisfação dos alunos com as atividades propostas, evidenciando a necessidade de aumentar as atividades práticas existentes nas disciplinas de ES. No entanto, o trabalho requeria o uso de ferramentas de desenvolvimento, teste de código fonte e não apresentava uma descrição detalhada da realização de cada atividade de forma separada. Diante do exposto, este trabalho visa avaliar as distintas metodologias e objetos de aprendizagem de baixa tecnologia utilizados em sala de aula no decorrer de uma disciplina de ES do ponto de vista dos discentes, a fim de obter mais informações quanto à preferência e o impacto dessas metodologias durante a disciplina. A seguir, são descritas as metodologias e objetos de aprendizagem aplicados e o seu processo de avaliação.

\section{Metodologias Aplicadas e Processo de Avaliação}

A disciplina de ES ocorreu no curso de Ciência da Computação da Universidade Federal do Maranhão - UFMA e foi realizada no segundo semestre de 2019. Nesta disciplina, foram aplicadas ao todo, 6 atividades/dinâmicas distintas em sala de aula, sendo elas: (1) Aulas Expositivas, (2) Dinâmica de Aviões para SCRUM [Duarte, 2018], (3) Aula Prática sobre Inspeção, (4) Prova com conteúdo considerando temas atuais, (5) Trabalho de Pesquisa, e (6) Aplicação de um jogo educacional em português na área de gerenciamento de riscos. A sugestão de aplicação destas atividades foi feita 
com base nos resultados obtidos no trabalho de Mendes et al. (2019), visando ter um balanceamento entre teoria e prática, além do uso de metodologias diferentes das tradicionais. A seguir, é explicada cada uma das atividades e seu processo de aplicação.

As Aulas Expositivas foram preparadas para permitir a apresentação dos conteúdos programáticos de acordo com a ementa da disciplina. Procurou-se dividir a aula em estágios de apresentação, visando: lembrar os conteúdos anteriores, associar os conteúdos com o tópico da aula em questão, introduzir os conceitos da aula corrente, explicar a importância e o impacto destes conceitos para um profissional na área de computação e fixar os conhecimentos através de um exercício prático. Cada aula expositiva teve uma duração de 60 minutos incluindo as etapas descritas acima, com exceção do exercício de fixação que era apresentado nos 40 minutos restantes, totalizando 1 hora e 40 minutos de aula. Vale ressaltar que o professor era responsável pela revisão dos exercícios de fixação e eles sempre eram resolvidos em sala de aula.

A Dinâmica de Aviões para SCRUM [Duarte, 2018] teve como objetivo a criação de uma linha de produção de aviões de papéis, permitindo ao aluno vivenciar os conceitos da metodologia ágil de desenvolvimento SCRUM de forma prática. Para isto, a turma foi dividida em equipes de aproximadamente 6 a 7 pessoas que prepararam aviões em papel utilizando uma guia de como dobrar o papel para construir um avião. Cada membro da equipe possuía uma atividade específica dentro do processo de desenvolvimento e o objetivo era utilizar conceitos relacionados ao SCRUM para melhorar a produtividade da equipe. A dinâmica induz problemas no processo de desenvolvimento que devem ser contornados e permitem a discussão do processo de desenvolvimento e o impacto de conceitos sobre gerenciamento de projetos na produtividade da equipe de desenvolvimento. Para esta dinâmica, foi utilizada uma aula de 1 hora e 40 minutos, sendo ela dividida em 3 etapas: apresentação (20 minutos), execução da parte prática (1 hora) e discussão (20 minutos).

A Aula Prática sobre Inspeção visou a realização de um processo de garantia da qualidade aplicado a artefatos do processo de análise de um sistema computacional fictício. Nesse sentido, artefatos de software para a especificação das funcionalidades de um sistema (diagrama de casos de uso e especificação de casos de uso) foram utilizados para aplicar a técnica de Inspeção de Descrições de Casos de Uso [Deboni e Gregolin, 2008]. Esta técnica possui um checklist com itens de verificação que permitem avaliar atributos como completeza, compreensibilidade, precisão, ambiguidade e consistência entre a especificação de casos de uso e o diagrama de casos de uso. Para o uso desta técnica, foi aplicado o processo proposto por Sauer et al. (2000) em que existe um treinamento, execução da inspeção de forma individual preenchendo um formulário (já disponibilizado pelos autores da técnica de inspeção), discussão dos defeitos identificados e correção deles. Este processo foi executado em 3 aulas de 1 hora e 40 minutos. Na primeira aula, foi executado o treinamento da técnica e feita a inspeção do artefato de forma individual. Na segunda aula, foi feita a discussão dos defeitos em grupos de 3 a 5 pessoas, e foram feitas modificações nos artefatos para atender os atributos de qualidade indicados pela técnica adotada. O tamanho das equipes foi proposto, considerando que o número de defeitos começa a se repetir a partir do aumento do número de inspetores no processo de inspeção [Sauer et al., 2000]. Finalmente, na terceira aula, ocorreu a discussão dos resultados obtidos, incluindo: defeitos identificados, discussão da equipe, correção de problemas e impressões sobre o uso da técnica de inspeção. 
$\mathrm{Na}$ Prova com conteúdo considerando temas atuais teve-se por objetivo incluir situações atuais e do cotidiano nas avaliações, como modelagem de sistemas de informação que resolvessem problemas gerados a partir de notícias em jornais e/ou programas de televisão. Com isso, esperava-se despertar o interesse do aluno em relação a como as metodologias ensinadas poderiam ser aplicadas na modelagem de sistemas contemporâneos. Para elaborar a prova, consultaram-se sites de notícias que tinham viralizado no contexto das redes sociais dos estudantes. Por exemplo, no contexto do programa de internet Pesadelo na Cozinha ${ }^{1}$, foram preparadas questões na prova que utilizaram estas informações para sua contextualização, e visavam resolver um problema relacionado ao contexto da notícia utilizando um sistema computacional. No contexto da notícia citada, a prova solicitava a modelagem de um sistema para gerenciamento da temperatura de um freezer inteligente, objeto da notícia relacionada ao programa de televisão.

Com relação ao Trabalho de Pesquisa, foram sorteados temas de diferentes processos de desenvolvimento de software inovadores que deveriam ser apresentados através da escrita e exposição de um artigo. Os temas foram selecionados a partir de tendências no desenvolvimento de software ágil na literatura, visando entender o que é utilizado na indústria e como [Hoda et al., 2018]. Para esta atividade, os discentes contaram com duas aulas de 1 hora 40 minutos cada para discutir os tópicos de pesquisa selecionados e tirar dúvidas com o professor. Foi explicado o processo de realização de revisões da literatura em bibliotecas digitais, assim como o processo de escrita de um artigo relatando os resultados da revisão. As apresentações ocorreram em uma aula de 1 hora e 40 minutos em que os discentes foram sorteados para apresentar os resultados da revisão da literatura realizada, indicando o modelo de ciclo de vida de desenvolvimento de software pesquisado, e como este tinha sido adotado na prática, citando artigos científicos relacionados com o tópico escolhido.

Finalmente, com relação ao uso de um Jogo de Ensino de ES, foi aplicado o jogo Arriscando que trata do tópico de gestão de riscos [Santos et al., 2019]. Arriscando é um jogo de baralho não digital baseado em uma dinâmica de ataque (o surgimento de riscos em projetos de desenvolvimento de software) e defesa (o uso de mitigações adequadas para estes riscos) para abordar os imprevistos que podem ocorrer em um projeto de desenvolvimento de software. O objetivo do jogo é apresentar aos participantes as categorias de riscos, riscos existentes dentro destas categorias e as mitigações que podem ser usadas para diminuir o impacto negativo dos riscos nos projetos. $\mathrm{O}$ jogo permite fazer o relacionamento existente entre categorias de riscos e riscos, assim como riscos e mitigações. Com isso, o jogo visa o entendimento do impacto que os riscos podem ocasionar nos projetos de software e a importância de realizar planos de gerenciamento de riscos. Este jogo foi escolhido devido ao tópico que ele aborda (Gerenciamento de Riscos), sendo este um dos últimos tópicos de ensino da disciplina, e porque o jogo estava completo e disponível em idioma português. O jogo foi aplicado em uma aula de 1 hora e 40 minutos de duração em equipes de 3 a 5 pessoas (número de jogadores sugeridos pelos autores do jogo). Nesta aula, ocorreu a explicação de uma introdução ao conceito de riscos em projetos de desenvolvimento de

\footnotetext{
${ }^{1} \mathrm{https}$ //hugogloss.uol.com.br/tv/restaurante-que-desligava-o-freezer-a-noite-empesadelo-na-cozinha-e-colocado-a-venda-e-dono-se-surpreende-com-propostas-entendamotivo/
} 
software, o jogo e suas regras (30 minutos). Logo, ocorreu a aplicação do jogo propriamente dito (1 hora) e o encerramento da dinâmica com uma conclusão sobre gestão de riscos em projetos de software (10 minutos).

Para a avaliação das atividades adotadas em sala de aula, foi aplicado um questionário por meio do Google Forms composto por perguntas abertas e fechadas baseadas no trabalho de Braga (2015), a fim de coletar informações a serem analisadas de forma quantitativa e qualitativa. Além disso, foi realizada a dinâmica Focus Group Lovers VS Haters [França et al., 2015], em que os participantes são convidados a experimentar, opinar, debater e se posicionar em relação ao tema da pesquisa. A metodologia Focus Group - Lovers VS Haters é dividida em duas partes. A primeira é uma atividade de interpretação de papéis, em que o grupo é dividido em dois: "Lovers", que deverão argumentar a favor do objeto de estudo; e "Haters", que terão que construir argumentos contra o objeto de estudo. O segundo momento do Focus Group - Lovers VS Haters não é mais uma atividade de interpretação de papéis, e sim um momento em que todos terão a liberdade para discutir os pontos levantados durante o levantamento de argumentos e sugerir melhorias para o objeto de estudo. A avaliação permitiu a compreensão do grau de aceitação dos alunos na aplicação de atividades educacionais no ensino de ES.

\section{Resultados e Discussão}

A turma da disciplina de ES tinha, ao todo, 18 alunos. Os discentes da turma tinham idades entre 19 e 23 anos, e estavam cursando, na sua maioria, o quarto período do curso de Ciência da Computação da Universidade Federal do Maranhão. Nenhum dos discentes tinha cursado a disciplina de Engenharia de Software anteriormente e todos tinham pouco ou nenhum conhecimento da área de ES. Destes discentes, apenas 16 responderam o questionário, mas somente 10 alunos participaram de todas as atividades. Para que houvesse concordância entre o número de notas dadas pelos discentes para cada atividade de ensino adotada, foram consideradas apenas as respostas dos 10 discentes que participaram de todas as atividades e podiam, consequentemente, avaliar todas elas.

Com relação às questões abertas, para obter as percepções dos alunos em relação à cada atividade, foram criadas sete afirmativas para serem respondidas utilizando uma escala Likert de 5 pontos (concordo fortemente, concordo parcialmente, nem concordo nem discordo, discordo parcialmente e discordo fortemente). As afirmativas do questionário foram baseadas no trabalho de Braga (2015) para avaliação de objetos de aprendizagem: Item 1) Os conteúdos foram abordados de maneira clara e precisa; Item 2) A experiência foi relevante para o meu aprendizado; Item 3) Acredito que o conteúdo está atualizado; Item 4) A atividade possibilitou interação entre os participantes; Item 5) Durante a realização da atividade foram propostos desafios; Item 6) Eu recebi feedback das atividades realizadas; e Item 7) Eu gostaria de ter outras experiências com este tipo de atividade. Os resultados dessas percepções são apresentados na Figura 1. Como pode ser observado, as atividades com melhor avaliação nos itens do questionário (concordância forte ou parcial) foram: Dinâmica de Aviões para SCRUM, Aula Prática de Inspeção e Jogo de Ensino de ES. Estas atividades são as que permitiram maior grau de interação entre os discentes além de utilizarem metodologias e artefatos diferenciadas de ensino. Para melhor entender os aspectos positivos, negativos e 
oportunidades de melhoria destas abordagens, são apresentados a seguir, os resultados das questões abertas do questionário e a dinâmica de Focus Group - Lovers VS Haters.

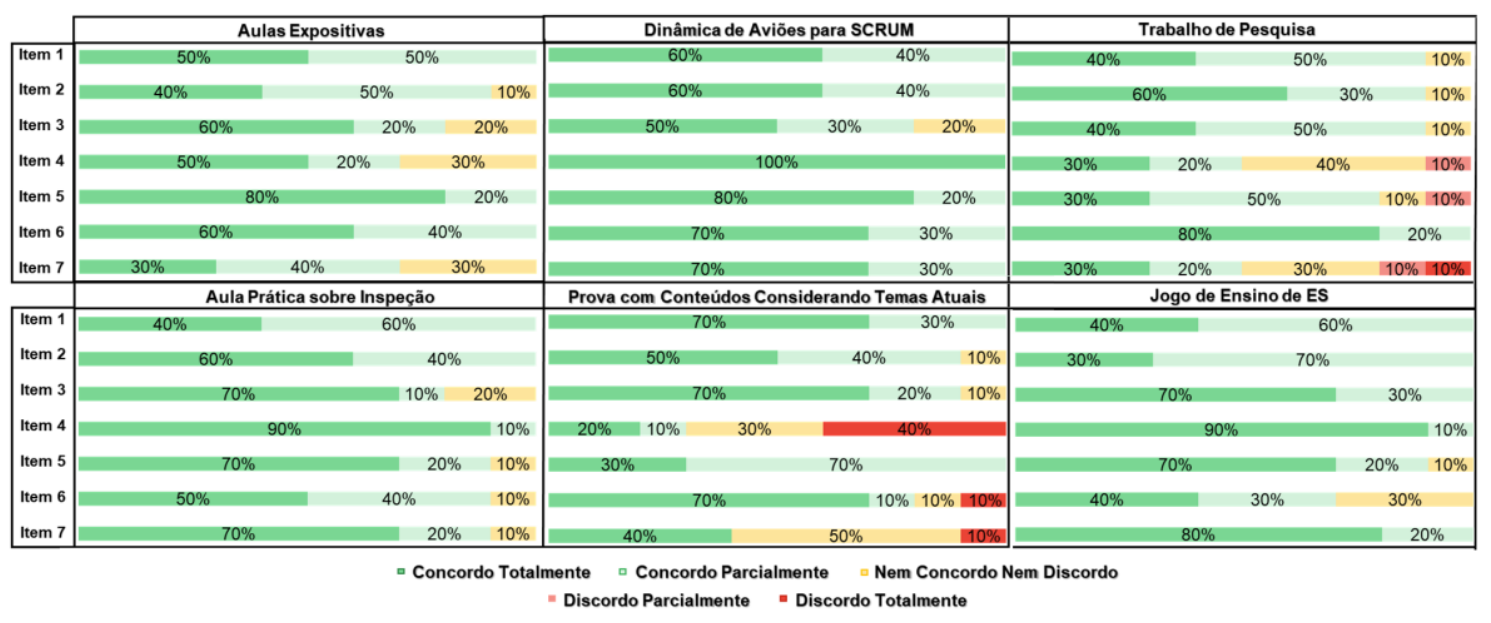

Figura 1. Avaliação das atividades educacionais aplicadas

As questões abertas possibilitaram que os alunos descrevessem os pontos positivos e negativos de cada atividade. Em relação às aulas expositivas, foram citados pontos positivos como: (i) boa didática na apresentação dos conteúdos, (ii) verificação de aprendizagem através dos exercícios ao final das aulas e (iii) espaço para exposição de opiniões e interação da turma; e como pontos negativos foi citado o excesso de conteúdo. A dinâmica de aviões para Scrum: (i) mostrou na prática o processo de desenvolvimento, (ii) possibilitou trabalho em equipe e (iii) ajudou na contextualização do conteúdo através da prática; porém, os alunos citaram a dificuldade durante a execução da dinâmica por conta da falta de uma apresentação do principal objetivo da atividade no início. A aula prática sobre inspeção: (i) estimulou os alunos na busca de soluções diante de falhas, (ii) os condicionou na inspeção de artefatos, e (iii) ajudou no acompanhamento do fluxo de trabalho. Em contrapartida, houve (i) pouco tempo para reelaboração dos artefatos e (ii) o material disponibilizado foi muito extenso. Por sua vez, a prova com temas atuais foi considerada positiva por ter (i) questões condizentes com os assuntos abordados em sala de aula, (ii) tópicos contextualizados com situações que puderam ser facilmente relacionadas com o mundo real e (iii) questões detalhadas e de cunho prático. O (i) pouco tempo para execução da prova e (ii) as questões longas que dependiam de bastante leitura foram os pontos negativos citados sobre a atividade. O trabalho de pesquisa: (i) possibilitou a descoberta de tópicos não apresentados em sala de aula, (ii) deu acesso a outro meio de aprendizado e à produção acadêmica da disciplina, além de (iii) permitir diferentes visões e conhecimento mais aprofundado sobre o tema. Todavia, os alunos levantaram que houve: (i) dificuldade na escrita do artigo devido à pouca experiência e conhecimento prévio dos conteúdos, considerando assim o (ii) trabalho muito extenso em relação ao prazo e ao número de alunos por equipe, além de apresentarem (iii) dificuldade na proposição de ideias que não tivessem sido apresentadas anteriormente. Por fim, o jogo aplicado: (i) possibilitou a fixação do conteúdo, (ii) estimulou o pensamento para resolução dos riscos, além de ser considerado um (iii) jogo intuitivo, atrativo e divertido. Como pontos fracos foi citado a (i) possibilidade de ganhar o jogo decorando as respostas, (ii) a falta de mitigações que adequadas para solução de eventuais riscos e a (iii) possibilidade de desfoque do conteúdo ao estar próximo da vitória no jogo. 
Em relação à dinâmica de Focus Group - Lovers VS Haters, foram feitas três afirmações quanto ao processo de ensino na disciplina de um modo geral. Esta dinâmica ocorreu no último dia de aula e teve uma duração de 1 hora e 40 minutos. As afirmações utilizadas durante a dinâmica foram: as atividades educacionais são aplicáveis para a disciplina; as atividades educacionais são uteis no processo de ensino/aprendizagem; e as atividades educacionais facilitam o processo de ensino aprendizagem. No quadro que afirmava que as atividades educacionais são aplicáveis para a disciplina, foram apresentados cartões com argumentos que diziam que as atividades: auxiliam no desenvolvimento de uma aplicação que vai além de programação, envolvendo outras áreas com processos diferentes; quebram a monotonia de uma aula totalmente expositiva; e promovem resultados mais eficientes quanto ao desenvolvimento do aluno. Contudo, os Haters afirmaram que as metodologias adotadas muitas das vezes fizeram com que a disciplina se tornasse cansativa, despertando o desinteresse e a desmotivação dos alunos. No quadro que diz respeito à utilidade das atividades educacionais, os Lovers afirmaram que elas foram úteis por oferecer um conhecimento que melhora a ética de trabalho do programador, além de permitir uma visão breve do que esperar da carreira de profissionais de TI. Porém, os Haters afirmaram que algumas atividades não permitem uma aprendizagem real antes da obtenção de uma boa base do conteúdo. Por fim, no quadro que afirmava que é fácil aprender utilizando diferentes metodologias, foi apresentado como argumento positivo pelos Lovers que as atividades ajudaram a fixar os conteúdos de maneira atrativa e divertida, pois a prática facilitou no processo de contextualização das teorias abordadas. Em contrapartida, os Haters relataram a falta de material didático atualizado para a execução de algumas atividades educacionais.

Considerando os aspectos indicados pelos discentes no questionário e na dinâmica de Focus Group - Lovers VS Haters, foi possível identificar lições aprendidas desta disciplina que podem ser consideradas em futuras reaplicações desta experiência. Ao planejar as disciplinas relacionadas à ES, os professores precisam determinar metas e objetivos, sobre o que vão ensinar e como conduzir a disciplina por meio destas metodologias. Diante dessas preocupações, é interessante ter conhecimento, por parte dos docentes, sobre as principais necessidades dos alunos em relação à aprendizagem de ES. Portanto, investigar os interesses dos alunos quanto aos conteúdos de ES se torna relevante. A partir disso, os docentes devem fazer um esforço para alinhar os conteúdos das ementas com os interesses dos discentes no contexto de questões tecnológicas e com à realidade do mercado de TI da sua localidade. Além disso, aspectos como: interação da turma; associação da atividade e do conteúdo estudado com a realidade local; uso de metodologias descontraídas; e associação do conteúdo com a realidade do mercado de software devem ser considerados no processo de ensino, de modo a obter avaliações positivas das atividades realizadas durante a disciplina por parte dos discentes.

\section{Conclusão e Trabalhos Futuros}

De modo geral, todas as atividades educacionais impactaram de forma positiva no processo de aprendizagem. Ao questionar os discentes sobre qual é a atividade preferida deles, as atividades foram ranqueadas na seguinte ordem: Dinâmica de Aviões para SCRUM, Jogo Educacional para ES, Aula Prática sobre Inspeção, Aulas Expositivas, Prova com Temas Atuais e Trabalho de Pesquisa. Segundo os discentes, as atividades que permitem interagir de forma divertida e aprender por meio da prática engajam mais do que atividades que focam no estudo individual e/ou pesquisar em outras fontes. 
Contudo, deve-se ressaltar que as atividades melhor avaliadas focaram na aprendizagem de conceitos e não necessariamente na sua aplicação, com exceção da aula prática sobre inspeção, que ficou em terceiro lugar. Portanto, deve-se procurar um meio de dinamizar as outras atividades para despertar o interesse dos discentes e alcançar os objetivos pedagógicos mais complexos do processo de ensino, como: aplicação, análise, síntese e avaliação de conceitos [Ferraz et al., 2010]. Vale ressaltar que as atividades aplicadas utilizaram materiais de baixa tecnologia, como papel e modelos para a construção de aviões, formulários, modelos de software impressos, artigos científicos, notícias disponíveis, entre outros, o que pode permitir a reaplicação destas metodologias em outros cenários de ensino sem o uso de computador.

Como trabalho futuro, tem-se em vista a reaplicação desse estudo em outras turmas, levando em consideração os pontos a serem melhorados de cada atividade educacional, além de incluir novas metodologias, abordagens e objetos de aprendizagem de baixa tecnologia no contexto de ensino de ES que não foram avaliados nesse momento. Além disso, pretende-se analisar o perfil dos alunos durante sua formação e após sua formatura, visando identificar de que forma as capacitações em ES adquiridas por eles durante a graduação interferem no desenvolvimento profissional dentro do mercado de trabalho. Com isso, os conteúdos ministrados e as metodologias adotadas podem ser adaptados para atender melhor as necessidades correntes do mercado de trabalho com relação ao ensino de computação na área de ES.

\section{Referências}

ACM/IEEE-CS Joint Curriculum Task Force Report. Computing Curricula 2013. Disponível online em: http://www.acm.org/education/curricula-recommendations.

Andrade, R. M. C., Santos, I. S., and Linhares, I. Uma metodologia para o ensino teórico e prático da engenharia de software. FEES 2015, page 60.

Andrade, R. M. C., Santos, I. S., Araújo, I. L., Aragão, B. S., \& Siewerdt, F. (2017, September). Retrospective for the Last 10 years of Teaching Software Engineering in UFC's Computer Department. In Proceedings of the 31st Brazilian Symposium on Software Engineering (pp. 358-367).

Araujo, N., Machado, R., Viana, D., and Rivero, L. (2017). Avaliando a viabilidade do blackbox em sala de aula: Um jogo sério para ensino de teste funcional de software. In Brazilian Symposium on Computers in Education (Simpósio Brasileiro de Informática na Educação - SBIE), volume 28, page 817.

Braga, J. C. (2015). Objetos de aprendizagem, volume 2: metodologia de desenvolvimento. Santo André: Editora da UFABC.

Cunha, J. A. O., Marques, G. A., Lemos, W. L., Câmara Jr, U. D., and Vasconcellos, F. J. (2018). Software engineering education in brazil: a mapping study. In Proceedings of the XXXII Brazilian Symposium on Software Engineering, pages 348-356.

Deboni, J. E. Z., \& Gregolin, R. (2008). Inspeção de Qualidade em Descrições de Casos de Uso: Uma Proposta de Modelo e Artefatos. VII SIMPÓSIO BRASILEIRO DE QUALIDADE DE SOFTWARE, 157.

Duarte, L. (2018) “Como ensinar Scrum na prática \#2 - LuizTools". https://www.luiztools.com.br/post/como-ensinar-scrum-na-pratica-2/. Dezembro. 
Ferraz, A. P. D. C. M., \& Belhot, R. V. (2010). Taxonomia de Bloom: revisão teórica e apresentação das adequações do instrumento para definição de objetivos instrucionais. Gestão \& Produção, 17(2), 421-431.

França, B. B. N., Ribeiro, T. V., Santos, P. S. M., \& Travassos, G. H. (2015). Using Focus Group in Software Engineering: lessons learned on characterizing software technologies in academia and industry. In CIbSE (p. 351).

Hoda, R., Salleh, N., \& Grundy, J. (2018). The rise and evolution of agile software development. IEEE software, 35(5), 58-63.

Humphrey, W. S. (1988, April). The software engineering process: definition and scope. In Proceedings of the 4th international software process workshop on Representing and enacting the software process (pp. 82-83).

Lemos, W., Cunha, J., \& Saraiva, J. (2019, July). Ensino de Engenharia de Software em um Curso de Sistemas de Informação: Uma Análise dos Problemas e Soluções na Perspectiva de Professores e Alunos. In Anais do XXVII Workshop sobre Educação em Computação (pp. 305-318). SBC.

Lima, J. V., Júnior, M. D. M. A., Moya, A., Almeida, R., Anjos, P., Lencastre, M., ... \& Alencar, F. (2019, November). As Metodologias Ativas e o Ensino em Engenharia de Software: uma revisão sistemática da literatura. In Anais do Workshop de Informática na Escola (Vol. 25, No. 1, p. 1019).

Mendes, J., Costa, Y., Frazão, K., Santos, R., Santos, D., and Rivero, L. (2019). Identificação das expectativas e dificuldades de alunos de graduação no ensino de engenharia de software. In Anais do XXVII Workshop sobre Educação em Computação, pages 334-347, Porto Alegre, RS, Brasil. SBC.

Santos, G., Rocha, A. R., Conte, T., Barcellos, M. P., \& Prikladnicki, R. (2012, September). Strategic alignment between academy and Industry: a Virtuous Cycle to Promote Innovation in Technology. In 2012 26th Brazilian Symposium on Software Engineering (pp. 196-200). IEEE.

Santos, R. E., Magalhães, C. V., Correia-Neto, J. S., \& Vilar, G. (2014). Ensino guiado por estudo dirigido: uma experiência no contexto da Engenharia de Software. In Anais do Workshop de Informática na Escola (Vol. 20, No. 1, p. 170).

Santos, S., Carvalho, F., Costa, Y., Viana, D., \& Rivero, L. (2019). Risking: A Game for Teaching Risk Management in Software Projects. In Proceedings of the XVIII Brazilian Symposium on Software Quality (pp. 188-197).

Sauer, C., Jeffery, D. R., Land, L., \& Yetton, P. (2000). The effectiveness of software development technical reviews: A behaviorally motivated program of research. IEEE Transactions on Software Engineering, 26(1), 1-14.

Wangenheim, C. G. and Silva, D. A. (2009). Qual conhecimento de engenharia de software é importante para um profissional de software? Proceedings of the Fórum de Educação em Engenharia de Software, 2:1-8.

Zorzo, A. F., Nunes, D. J., Matos, E. D. S., Steinmacher, I. F., Leite, J. C., De Araujo, R. M., Correia, R. C. M., and Martins, S. D. L. (2017). Referenciais de formação para os cursos de graduação em computação. 